\title{
The Determinants of Rating Announcements Impact on Stock Markets during Crisis Periods: The Case of the 2008 Worldwide Financial Crisis
}

\author{
Dorsaf Azouz Ghachem ${ }^{1}$ \\ ${ }^{1}$ Higher School of Economics and Commercial Sciences of Tunis, ESSEC Tunis, Department of Finance, DEFI \\ Research Unit, Tunis \\ Correspondence: Dorsaf Azouz Ghachem, Higher School of Economics and Commercial Sciences of Tunis, \\ ESSEC Tunis, Department of Finance, DEFI Research Unit, 4, Rue Abou Zakaria El Hafsi, 1089 Montfleury \\ Tunis. Tel: 216-2048-3035. E-Mail: dorsafazouz@yahoo.fr
}

Received: October 27, 2014

Accepted: January 13, 2015

Online Published: March 25, 2015

doi:10.5539/ijef.v7n4p159

URL: http://dx.doi.org/10.5539/ijef.v7n4p159

\begin{abstract}
Identifying the determinants of rating impact on stock markets during crisis periods allows on the one hand, explaining investors' behavior towards rating agencies, and on the other hand expecting their reaction to a rating announcement. This research reviews factors previously studied and adds three new ones: the announcement anticipation, the double rating and the foreign investors' presence in firm' shareholding. We use an event study methodology to determine the short term impact of rating announcements of 207 U.S. firms during the 2008 worldwide financial crisis. Significant reaction is measured by the cumulative abnormal return of the stock price after the rating announcement. Then, we regress the measured cumulative return on several factors related to the rating change and the rated firm. Results show that a bad rating impact on stock markets during crisis period is influenced by the anticipation of the announcement, the downgrade magnitude and firms' sizes. Good rating announcements are conditioned by the initial rating level, the upgrade magnitude and firms' sectors. Reaction to assertions only depends on anticipation and institutional presence.
\end{abstract}

Keywords: crisis, determinants, event study, rating impact

\section{Introduction}

Identifying the determinants of rating announcements on financial markets allows on the one hand explaining investors' behaviour towards rating agencies, and on the other hand expecting their reaction to a rating announcement.

Previous researches showed that investors tend to overreact to bad rating announcements during crisis period and to neglect good and neutral ones (Griffin \& Sanvicente, 1982; Hand Holthausen \& Leftwich, 1992; Boudriga, Azouz, \& Mamoghli, 2011). Specifically, they react more intensively to bad and neutral expected announcements. On the contrary, good news have a more important impact when they are unexpected. Otherwise, the determinants of investors' reaction to rating announcements on stock markets have not been exhaustively studied. In fact, previous researches focused only on the influence of few factors on rating impact.

As far as we know, this research is the first one to consider all the determinants that have been studied in previous researches. Moreover, this paper considers three additional factors that could be powerful during crisis period. These new factors are the announcement anticipation, the notation type (double or single rating) and foreign investors' presence in firms' shareholdings.

First, we focus on anticipation factor. We previously found that reaction to rating announcements changes whether they are expected or not (Hand et al., 1992; Purda, 2007; Boudriga \& Azouz, 2013). Indeed, difference of reaction between expected and surprise rating is of 1 day for neutral announcement. It becomes of 4 days after bad announcements. As regards good rating news, a significant difference is observed 1 day before the announcement. Investors react more to surprise upgrades than expected ones. Considering the investors' faith loss towards rating agencies, it seems that investors react more intensively to expected announcements that they have well studied than surprise changes. 
Second, we consider the influence of double rating on investors' decisions. Investors know that double notation alleviates the conflicts of interests between rating agencies and rated firms. Therefore, double ratings have more impact than single ones (Micu, Remonola, \& Wooldridge, 2004).

Finally, we introduce the foreign investors' presence in firms' shareholdings as a potential determinant due to the asymmetry information between local and foreign investors. The latter(s) lack informations as compared to local investors. Consequently, they tend to react intensively to rating news, which causes significant impacts on stock prices.

We use an event study methodology to determine the short term impact of rating announcements of 207 U.S. firms during the 2008 worldwide financial crisis. Significant reaction is measured by the cumulative abnormal return (CAR) of the stock price after the rating announcement. Then, we regress the measured cumulative return on several factors related to the rating change and the rated firm.

Our results show that the impact of bad rating news during the crisis period is influenced by the announcement anticipation, the rating magnitude and firms' size. Good announcements are conditioned by the initial rating level and firms' sector. Reactions to assertions depend only on anticipation criterion.

The remainder of the paper is organized as follows. Section 1 reviews literature on explanatory factors of rating impact. Section 2 develops hypotheses related to rating impact determinants. Section 3 describes the methodology and data employed. Section 4 provides empirical results while section 5 concludes.

\section{Literature Review}

Existing literature shows ambiguous results related to the determinants of market reaction to rating announcements during stable periods.

Hand et al. (1992) indicate that the impact of rating announcements on bond and stock prices depends on the initial rating level, the change of rating class (speculative/ investment grade), the rating magnitude and the period separating two successive ratings. Schweitzer, Szewczyk and Varma (1992) show that financial and banking securities are more sensible to rating announcements than non financial ones. In fact, financial and bank institutions do not reveal bad news in order to maintain order and stability on markets. Consequently, rating agencies provide investors with bad unknown news that generate strong negative impact on prices. These results corroborate those of Gropp and Richards (2001) and Bonini, Pettinato and Salvi (2009). However, they are in contradiction with those of Sunder (1991) who highlights that rating impact on stock markets is independent of any factor. Ederington and Goh (1993) show that the origin of rating change influences market reaction. Unlike rating changes caused by the deterioration of financial indicators which are rejected by the market, rating changes resulting from the firm leverage increase are well perceived by stock markets. Nayar and Rozeff (1994) corroborate these results. In fact, authors found that rating impact on stock markets is influenced by the initial rating level and the firm level leverage. Dichev and Piotroski (2001) and John, Ravid and Reisel (2005) point out that market reaction to rating announcements is dependent upon these factors and also the firm size and status (holding or subsidiary). Micu et al. (2004) work on the rating impact on CDS prices. They found that double and triple ratings have higher impact than single ones. Also, investors' reactions are tenacious when securities are speculative and belong to small firms. Halek and Eckles (2010) advance that bad news generate stronger reaction than good ones. Reaction depends on downgrade magnitude and institutional investors' presence in firms' shareholdings. Creighton et al. (2007) study the impact of rating announcements on Australian market. They find that ratings impact especially small and low rated firms. Linciano (2008) finds that Italian stock market intensely responds to ratings of financial firms. Avramov, Chordia, Jostova and Philipov (2009) make clear that the determinants of the rating impact on stock prices are firm size, leverage, financial performance, presence of institutional investors on shareholding, rating magnitude and the initial level of rating.

Announcement anticipation, rating type and presence of foreign investors in shareholding, have not yet been considered as determinants of rating impact on stock markets. Hand et al. (1992) and Di Cesare (2006) find that investors react differently to rating announcements whether they are expected or not. Theoretically, surprise announcements have greater effects on stock prices than expected ones. Indeed, they vehicle unknown informations to investors. On the contrary, Purda (2007) highlights the same impact for both rating types. Consequently, the anticipation influence on rating impact deserves to be studied during crisis period.

Considering rating type, Raimbourg (1990) demonstrates that double ratings are more credible than single ones. In fact, investors are aware of interest conflicts between rating agencies and firms. Due to paid fees, rating agencies tend to be complacent in rating firms. Therefore, double ratings allow alleviating these conflicts and having more impact on stock prices than single ones. 
Finally, Frankel and Schmukler (1996, 1998a) advance that asymmetry information makes foreign investors more conservative when making their investment decisions. However, refer to Karolyi (2002); foreign presence has no effect on stock prices by cause of the small foreign participation in firms' shareholding.

\subsection{Hypotheses}

Taking into account the above literature review, we will work in the empirical part of the paper with the following hypotheses.

(1) Investors during crisis period respond more intensively to expected announcements than surprise ones.

(2) Investors care more about speculative than investment grade securities during crisis period.

(3) The impact of rating announcements on stock prices is greater when it changes securities from investment to speculative grade or vice versa.

(4) Investors' reaction to rating announcements during crisis period is an increasing function of the rating change magnitude.

(5) Double ratings have stronger effects on stock prices than single ones.

(6) Rating announcements of financial securities have more impact on stock prices than non financial ones.

(7) Investors respond to big firm ratings more than they do to small ones due to potential losses incurred by big firms.

(8) The most indebted firms ratings have greater impact on stock prices than less leveraged ones.

(9) Investors' reaction to rating announcements during crisis period is an increasing function of firm financial performance.

(10) Rating impact on stock price is an increasing function of institutional investors' presence in shareholding.

(11) Rating effect on stock price is an increasing function of foreign investors' presence in shareholding.

\section{Data and Methodology}

\subsection{Data}

We collect 207 rating announcements from Moodys and Standard and Poors. The period extends from $16^{\text {th }}$ September to $31^{\text {st }}$ December 2008. It coincides with the beginning of the 2008 worldwide financial crisis. We separate between bad news such as downgrades and revisions to downgrades, good news such as upgrades and revisions to upgrades and assertions or neutral news. Our data sources are the agencies websites and Compustat North America database.

Table 1. Descriptive statistics of the sample

\begin{tabular}{|c|c|c|c|}
\hline News & $\mathrm{Bad}$ & Good & Assertions \\
\hline Number & 141 & 14 & 52 \\
\hline$\%$ & 68.1 & 6.8 & 25.1 \\
\hline Total & \multicolumn{3}{|c|}{207} \\
\hline
\end{tabular}

The firms sample contains different sectors: energy, textile, chemical, automobile, metallurgy, food and catering, health, technology, telecommunications, property development, services including transport, advertising and various consumption (leisure, drugstore, and cosmetics) and financial activities such as banks, insurance and investment companies.

Table 2. Businesses' firms of the sample

\begin{tabular}{lllll}
\hline Businesses / News & Bad & Good & Neural \\
\hline \multirow{4}{*}{ Industry } & Energy (\%) & $6(4.3)$ & $2(14.3)$ & $3(5.7)$ \\
& Textile (\%) & $5(3.5)$ & $0(0)$ & $0(0)$ \\
& Chemistry (\%) & $1(0.7)$ & $0(0)$ & $2(3.8)$ \\
& Automotive (\%) & $6(4.3)$ & $0(0)$ & $1(1.9)$ \\
& Other (\%) & $9(6.4)$ & $2(14.3)$ & $8(15.5)$ \\
\hline
\end{tabular}




\begin{tabular}{|c|c|c|c|c|}
\hline \multicolumn{2}{|c|}{ Metallurgy (\%) } & $4(2.8)$ & $1(7.1)$ & $1(1.9)$ \\
\hline \multicolumn{2}{|c|}{ Food and Catering (\%) } & $14(9.9)$ & $0(0)$ & $1(1.9)$ \\
\hline \multicolumn{2}{|c|}{ Health $(\%)$} & $8(5.7)$ & $2(14.3)$ & $3(5.7)$ \\
\hline \multicolumn{2}{|c|}{ Technology and Telecommunications (\%) } & $13(9.2)$ & $1(7.1)$ & $7(13.5)$ \\
\hline \multicolumn{2}{|c|}{ Real Estate (\%) } & $6(4.2)$ & $0(0)$ & $1(1.9)$ \\
\hline \multicolumn{2}{|c|}{ Services (Transportation, Advertising) (\%) } & $7(5)$ & $1(7.1)$ & $2(3.8)$ \\
\hline \multicolumn{2}{|c|}{ Various consumption (Leisure, drugstore, cosmetics) (\%) } & $16(11.3)$ & $2(14.3)$ & $2(3.8)$ \\
\hline \multirow{3}{*}{ Finance } & Bank (\%) & $10(7)$ & $2(14.3)$ & $2(3.8)$ \\
\hline & Insurance $(\%)$ & $4(2.8)$ & $0(0)$ & $0(0)$ \\
\hline & Investment $(\%)$ & $32(22.9)$ & $1(7.1)$ & $19(36.8)$ \\
\hline \multicolumn{2}{|l|}{ Total } & 141 & 14 & 52 \\
\hline
\end{tabular}

Table 3. Descriptive statistics of firms

\begin{tabular}{llllll}
\hline Thousands $\$$ & Mean & Median & Maximum & Minimum & Standard Error \\
\hline Total Assets & 88813,7 & 5870,5 & 2187631 & 299,6 & 309454,6 \\
Total liabilities & 81640,2 & 3626,3 & 2074033 & 99,8 & 292758 \\
Long terme debt & 17661,2 & 1152,7 & 427112 & 0 & 59203 \\
Equity & 7170,3 & 1568,2 & 133176 & -56970 & 19104,7 \\
Net income & 58,9 & 17,8 & 8915 & -15471 & 1953 \\
\hline
\end{tabular}

\subsection{Methodology}

First, we use an event study to measure the market reaction to rating news. It consists of calculating stock cumulative abnormal return before and after a rating announcement. The event window extends on 20 days symmetrically set around the day announcement (day 0). Cumulative abnormal returns are calculated by two models: the stock index adjusted model and the market adjusted model.

Moreover, rating announcements are subdivided according to whether they are expected or not. Expected announcements are preceded by a significant cumulative abnormal return on 120 days preceding the day announcement (Di Cesare, 2006).

\subsubsection{Construction of Variables}

Endogenous variable is the stock cumulative abnormal return (noted car) calculated on 10 days succeeding the announcement. The following explanatory variables are retained:

- $\quad$ The announcement anticipation (noted ant): a binary variable equal 1 if the announcement is expected, 0 otherwise.

- $\quad$ The initial level of rating (noted nin): a binary variable equal 1 if the equity is speculative, 0 otherwise.

- $\quad$ Class change of rating (noted cc1): a binary variable equal 1 if the announcement changes the equity rating class from investment to speculative or vice versa, 0 otherwise

- $\quad$ Rating change magnitude (noted amp): a quantitative variable calculated as the number of grades changed (new rating less old rating).

- $\quad$ Double notation (noted dn): a binary variable equal 1 if a firm is doubly rated by two agencies within a short space of time (Note 1), 0 otherwise.

- $\quad$ Firm business sector (noted sa): a binary variable equals 1 if the firm belongs to financial sector : banks, insurance or investment companies, 0 otherwise.

- $\quad$ Firm size (noted lntaille): a quantitative variable calculated as the natural logarithm of firm's total assets.

- $\quad$ Firm leverage (noted ne1): a quantitative variable calculated as the ratio of long term debt relative to total assets. To check the robustness of the leverage variable, we replace it in a second time by ne 2 representing the leverage ratio: total debt relative to equity.

- $\quad$ Financial performance (noted roe): a quantitative variable (return on equity) calculated as the ratio of net income to equity. To test the robustness of the financial performance, we replace it in a second time by $p b v$ (price book value). 
- Institutional investors' presence (noted nbre_ii): a quantitative variable calculated as the proportion of shares held by institutional investors. We replace it in a second time by nbre ii computed as the proportion in number of institutional investors.

- Foreign investors' presence (noted nbre ie): a quantitative variable calculated as the proportion of shares held by foreign investors. We replace it in a second time by nbre ie computed as the proportion in number of foreign investors.

\subsubsection{Explanatory Models}

Bad news: we use least ordinary squares regression to construct a linear model. Structural variables concern rating announcements. Control variables (noted fc) involve firms' characteristics (business, size, leverage, financial performance, institutional investors' presence, foreign investors' presence).

$$
C A R_{j}=\propto_{0}+\beta_{1} * \text { ant }+\beta_{2} * \text { nin }+\beta_{3}{ }^{*} \mathrm{cc}_{1(2)}+\beta_{4} * a m p+\beta_{5} * d n+\beta_{6} * f_{C}+\varepsilon
$$

We construct the variables correlation matrix and use the Variance Inflation Factors $(V I F)$ to detect whether collinearity exists between variables and heteroscedasticity between errors.

Good news: As regard the little size of good news sample, we just establish the correlation matrix and perform the Chi square independence test between the cumulative abnormal return and qualitative variables of ratings and firms. Otherwise, we calculate the Spearman's rank correlation coefficient to measure dependence between endogenous variable and quantitative explanatory variables.

Neutral news or assertions: we use least ordinary squares regression to construct a linear model. We omit some structural variables related to rating due to the neutral nature of assertions. These variables are: the magnitude (assertions have no magnitude) and the rating class change (assertion maintains security in the same class).

$$
C A R_{j}=\propto_{0}+\beta_{1}{ }^{*} \text { ant }+\beta_{2}{ }^{*} \operatorname{nin}+\beta_{3} * d n+\beta_{4}{ }^{*} f_{c}+\varepsilon
$$

\section{Empirical Results}

\subsection{Bad News}

The Variance Inflation Factors test invalids the presence of multicollinearity between independent variables. Also, the correlation matrix (Note 2) allows eliminating the existence of significant correlations between these variables.

Table 4. VIF test results: explanatory variables of market reaction to bad rating news during crisis period

\begin{tabular}{lcccccccc}
\hline Variables & ant & nin & $c c 1$ & $a m p$ & $d n$ & $s a$ & $p b v$ & $i i$ \\
\hline VIF & 1.43 & 1.90 & 1.14 & 1.23 & 1.19 & 1.99 & 1.27 & 1.51 \\
Variables & nbre_ii & ie & nbre_ie & $n e 1$ & $n e 2$ & lntaille & roe & MeanVIF \\
VIF & 1.69 & 2.23 & 2.54 & 1.29 & 1.11 & 2.07 & 1.29 & 1.59 \\
\hline
\end{tabular}

Note. ant: anticipation criterion, nin:rating initial level (speculative or investment grade); cc (1): rating change class (from investment grade to speculative); amp: change amplitude; dn: double rating; sa: business firm (financial and banking or not); pbv: price book value; ii: institutional investors' presence in shareholding in terms of monetary values; nbre_ii: institutional investors' presence in shareholding in terms of shareholders number; ie: foreign investors' presence in shareholding in terms of monetary values; nbre ie: foreign investors' presence in shareholding in terms of shareholders number; ne1: total debt / total assets; ne2: total debt / equity; lntaille: natural logarithm of total assets; roe: return on equity (Net income /equity).

In model I, we first regress the cumulative abnormal return on rating factors. In model II, we add the firm sector and size as control variables. We find that anticipation, magnitude and firm size remain significant. Also, results are the same when introducing other control factors, such as pbv (model III), roe (model IV), nel (model V) and ne2 (model VI), ii (model VII), nbre_ii (model VIII), ie (model IX), and nbre_ie (model X), and when replacing the endogenous variable $\mathrm{CAR}_{1}$ by $\mathrm{CAR}_{2}$ (Note3). 
Table 5. Correlation matrix of endogenous and exogenous variables relative to bad rating news during crisis period

\begin{tabular}{|c|c|c|c|c|c|c|c|c|c|c|c|c|c|c|c|c|c|c|}
\hline & carl & car2 & ant & $\operatorname{nin}$ & $c c 1$ & $c c 2$ & amp & $d n$ & $S a$ & $p b v$ & $i i$ & nbre_ii & ie & nbre-ie & nel & $n e 2$ & lntaille & roe \\
\hline carl & 1 & 0.07 & -0.07 & 0.07 & -0.03 & 0.09 & -0.08 & 0.08 & 0.12 & -0.05 & -0.32 & 0.08 & -0.06 & 0.33 & -0.02 & 0.09 & 0.27 & 0.16 \\
\hline car2 & & 1 & -0.15 & -0.04 & -0.09 & -0.06 & 0.03 & -0.01 & 0.19 & -0.12 & -0.03 & 0.02 & -0.10 & 0.05 & -0.07 & 0.04 & 0.22 & 0.12 \\
\hline Ant & & & 1 & 0.20 & 0.00 & 0.01 & 0.13 & 0.01 & -0.28 & -0.13 & -0.05 & 0.08 & 0.26 & 0.07 & 0.15 & -0.12 & -0.14 & -0.24 \\
\hline $\mathrm{Nin}$ & & & & 1 & -0.13 & 0.13 & 0.25 & 0.06 & -0.45 & -0.22 & -0.20 & -0.28 & -0.12 & -0.19 & 0.31 & -0.09 & -0.49 & -0.22 \\
\hline$c c 1$ & & & & & 1 & 0.33 & 0.16 & 0.07 & 0.02 & -0.10 & 0.08 & 0.01 & 0.03 & 0.00 & 0.09 & 0.06 & -0.03 & 0.03 \\
\hline$c c 2$ & & & & & & 1 & 0.21 & -0.02 & 0.08 & -0.10 & -0.04 & 0.07 & 0.09 & 0.12 & 0.10 & 0.15 & 0.05 & -0.02 \\
\hline$A m p$ & & & & & & & 1 & -0.19 & -0.13 & 0.01 & 0.06 & -0.07 & -0.01 & -0.06 & 0.21 & -0.00 & -0.17 & -0.00 \\
\hline Dn & & & & & & & & 1 & -0.10 & -0.11 & -0.14 & 0.04 & 0.10 & 0.02 & 0.13 & -0.08 & 0.05 & 0.08 \\
\hline$S a$ & & & & & & & & & 1 & -0.15 & -0.01 & 0.16 & 0.05 & 0.18 & -0.24 & 0.24 & 0.58 & 0.14 \\
\hline$P b v$ & & & & & & & & & & 1 & 0.14 & 0.11 & -0.07 & -0.07 & 0.03 & -0.02 & 0.00 & 0.07 \\
\hline$i i$ & & & & & & & & & & & 1 & 0.40 & -0.09 & -0.13 & -0.07 & -0.07 & 0.06 & 0.17 \\
\hline nbre_ii & & & & & & & & & & & & 1 & 0.22 & 0.35 & -0.03 & 0.02 & 0.33 & 0.06 \\
\hline ie & & & & & & & & & & & & & 1 & 0.68 & -0.02 & 0.00 & 0.13 & 0.12 \\
\hline nbre-ie & & & & & & & & & & & & & & 1 & -0.17 & 0.05 & 0.33 & 0.17 \\
\hline nel & & & & & & & & & & & & & & & 1 & -0.10 & -0.24 & -0.20 \\
\hline$n e 2$ & & & & & & & & & & & & & & & & 1 & 0.15 & -0.06 \\
\hline Intaille & & & & & & & & & & & & & & & & & 1 & 0.26 \\
\hline roe & & & & & & & & & & & & & & & & & & 1 \\
\hline
\end{tabular}

Note. CAR ( 1 2) cumulative abnormal return 4 days following the announcement, relative to stock adjusted model (1) or market adjusted model (2); ant: anticipation criterion, nin: rating initial level (speculative or investment grade); cc (1): rating change class (from investment grade to speculative); amp: change amplitude; dn: double rating; sa: business firm (financial and banking or not); pbv: price book value; ii: institutional investors' presence in shareholding in terms of monetary values; nbre ii: institutional investors' presence in shareholding in terms of shareholders number; ie: foreign investors' presence in shareholding in terms of monetary values; nbre_ie: foreign investors' presence in shareholding in terms of shareholders number; ne1: total debt / total assets; ne2: total debt / equity; lntaille: natural logarithm of total assets; roe: return on equity (Net income /equity).

Table 6. Bad news determinants during crisis period: results of CAR1 regression on rating characteristics with introduction of firm business and firm size

\begin{tabular}{lllll}
\hline & Model I & \multicolumn{3}{l}{ Model II } \\
\cline { 2 - 5 } Variables & Coef. & $P$-Val & Coef. & $P$-Val \\
\hline ant & $-0,152$ & $0,030^{* *}$ & $-0,142$ & $0,046^{* *}$ \\
nin & $-0,064$ & 0,369 & 0,028 & 0,729 \\
cc1 & $-0,171$ & 0,176 & $-0,140$ & 0,265 \\
amp & 0,045 & 0,223 & 0,047 & 0,197 \\
dn & $-0,004$ & 0,945 & $-0,010$ & 0,875 \\
sa & & & 0,045 & 0,621 \\
lntaille & & & 0,037 & $0,092^{*}$ \\
Const. & $-0,081$ & 0,240 & $-0,497$ & $0,023^{* *}$ \\
Adjusted $\mathrm{R}^{2}$ & 0,02 & & 0,05 & \\
Nb.Observations & 139 & & 139 & \\
\hline
\end{tabular}

Note. $* * *, * *$ and $*$ denote significance at $1 \%, 5 \%$ and $10 \%$.

Table 7. Bad news determinants during crisis period: results of CAR1 regression on rating characteristics with introduction of financial performance and indebtedness

\begin{tabular}{lllllllll}
\hline & Model III & \multicolumn{3}{c}{ Model IV } & Model V & \multicolumn{3}{c}{ Model VI } \\
\hline Variables & Coef. & $P$-Val & Coef. & $P$-Val & Coef. & $P$-Val & Coef. & $P$-Val \\
ant & $-0,154$ & $0,034^{* *}$ & $-0,141$ & $0,052^{*}$ & $-0,139$ & $0,053^{*}$ & $-0,148$ & $0,038^{* *}$ \\
nin & 0,003 & 0,971 & 0,028 & 0,726 & 0,040 & 0,625 & 0,032 & 0,691 \\
ccl & $-0,157$ & 0,215 & $-0,140$ & 0,267 & $-0,131$ & 0,298 & $-0,129$ & 0,304 \\
amp & 0,050 & 0,172 & 0,047 & 0,202 & 0,052 & 0,164 & 0,048 & 0,194 \\
dn & $-0,172$ & 0,800 & $-0,010$ & 0,874 & $-0,002$ & 0,970 & $-0,015$ & 0,814 \\
\hline
\end{tabular}




\begin{tabular}{|c|c|c|c|c|c|c|c|c|}
\hline sa & 0,018 & 0,845 & 0,045 & 0,619 & 0,040 & 0,655 & 0,061 & 0,502 \\
\hline lntaille & 0,038 & $0,088^{*}$ & 0,037 & $0,098 *$ & 0,037 & $0,010^{*}$ & 0,039 & $0,081 *$ \\
\hline pbv & $-0,023$ & 0,364 & & & & & & \\
\hline roe & & & 0,005 & 0,944 & & & & \\
\hline ne1 & & & & & $-0,127$ & 0,409 & & \\
\hline ne2 & & & & & & & $-0,002$ & 0,252 \\
\hline Const. & $-0,426$ & $0,065^{*}$ & $-0,495$ & $0,024 * *$ & $-0,466$ & $0,035^{* *}$ & $-0,505$ & $0,020^{*}$ \\
\hline Adjusted $\mathrm{R}^{2}$ & 0,05 & & 0,04 & & 0,04 & & 0,05 & \\
\hline Nb.Observations & 139 & & 139 & & 139 & & 139 & \\
\hline
\end{tabular}

Note. ${ }^{* * *}, * *$ and $*$ denote significance at $1 \%, 5 \%$ and $10 \%$.

Table 8. Bad news determinants during crisis period: results of CAR1 regression on rating characteristics with introduction of shareholding structure (institutional and foreign investors' presence)

\begin{tabular}{|c|c|c|c|c|c|c|c|c|}
\hline \multirow[b]{2}{*}{ Variables } & \multicolumn{2}{|c|}{ Model VII } & \multicolumn{2}{|c|}{ Model VIII } & \multicolumn{2}{|c|}{ Model IX } & \multicolumn{2}{|c|}{ Model X } \\
\hline & Coef. & $P$-Val & Coef. & $P-V a l$ & Coef. & $P$-Val & Coef. & $P$-Val \\
\hline ant & $-0,144$ & $0,044 * *$ & $-0,135$ & $0,061^{*}$ & $-0,116$ & 0,120 & $-0,141$ & $0,051 *$ \\
\hline $\operatorname{nin}$ & 0,016 & 0,839 & 0,017 & 0,829 & 0,015 & 0,845 & 0,027 & 0,737 \\
\hline $\mathrm{ccl}$ & $-0,135$ & 0,281 & $-0,140$ & 0,265 & $-0,140$ & 0,264 & $-0,140$ & 0,267 \\
\hline amp & 0,049 & 0,182 & 0,048 & 0,194 & 0,048 & 0,186 & 0,047 & 0,199 \\
\hline $\mathrm{dn}$ & $-0,016$ & 0,813 & $-0,008$ & 0,899 & $-0,001$ & 0,987 & $-0,010$ & 0,877 \\
\hline sa & 0,037 & 0,680 & 0,042 & 0,645 & 0,049 & 0,584 & 0,045 & 0,621 \\
\hline Intaille & 0,038 & $0,089 *$ & 0,041 & $0,072 *$ & 0,039 & $0,082 *$ & 0,038 & $0,095^{*}$ \\
\hline ii & $-0,099$ & 0,523 & & & & & & \\
\hline nbre_ii & & & $-0,290$ & 0,474 & & & & \\
\hline ie & & & & & $-0,181$ & 0,231 & & \\
\hline nbre_ie & & & & & & & $-0,028$ & 0,875 \\
\hline Const. & $-0,407$ & 0,116 & $-0,263$ & 0,500 & $-0,488$ & $0,025 * *$ & $-0,498$ & $0,023 * *$ \\
\hline Adjusted $\mathrm{R}^{2}$ & 0,04 & & 0,04 & & 0,05 & & 0,04 & \\
\hline Nb.Observations & 139 & & 139 & & 139 & & 139 & \\
\hline
\end{tabular}

Note. ${ }^{* *}, * *$ and $*$ denote significance at $1 \%, 5 \%$ and $10 \%$.

Table 9. Bad news determinants during crisis period: results of CAR2 regression on rating characteristics with introduction of firm business and firm size

\begin{tabular}{lllll}
\hline & \multicolumn{3}{c}{ Model I } & Model II \\
\cline { 2 - 5 } Variables & Coef. & $P$-Val & Coef. & $P$-Val \\
\hline ant & $-0,130$ & $0,088^{*}$ & $-0,111$ & 0,151 \\
nin & $-0,037$ & 0,633 & 0,074 & 0,400 \\
cc1 & $-0,165$ & 0,229 & $-0,132$ & 0,332 \\
amp & 0,036 & 0,372 & 0,038 & 0,334 \\
dn & 0,013 & 0,851 & 0,011 & 0,880 \\
sa & & & 0,092 & 0,347 \\
lntaille & & & 0,038 & 0,116 \\
Const. & $-0,042$ & 0,570 & $-0,495$ & $0,036^{* *}$ \\
Adjusted R & 0,00 & & 0,03 & \\
Nb.Observations & 139 & & 139 & \\
\hline
\end{tabular}

Note.***, ** and * denote significance at $1 \%, 5 \%$ and $10 \%$. 
Table 10. Bad news determinants during crisis period: results of CAR2 regression on rating characteristics with introduction of financial performance and indebtedness

\begin{tabular}{|c|c|c|c|c|c|c|c|c|}
\hline \multirow[b]{2}{*}{ Variables } & \multicolumn{2}{|c|}{ Model III } & \multicolumn{2}{|c|}{ Model IV } & \multicolumn{2}{|c|}{ Model V } & \multicolumn{2}{|c|}{ Model VI } \\
\hline & Coef. & $P-$ Val & Coef. & $P$-Val & Coef. & $P$-Val & Coef. & $P$-Val \\
\hline ant & $-0,132$ & $0,093 *$ & $-0,110$ & 0,162 & $-0,109$ & 0,159 & $-0,119$ & 0,124 \\
\hline $\operatorname{nin}$ & 0,030 & 0,741 & 0,074 & 0,401 & 0,080 & 0,374 & 0,079 & 0,365 \\
\hline $\mathrm{ccl}$ & $-0,163$ & 0,234 & $-0,132$ & 0,333 & $-0,128$ & 0,350 & $-0,118$ & 0,386 \\
\hline amp & 0,044 & 0,272 & 0,038 & 0,340 & 0,040 & 0,315 & 0,039 & 0,327 \\
\hline $\mathrm{dn}$ & $-0,000$ & 0,994 & 0,010 & 0,882 & 0,014 & 0,842 & 0,004 & 0,956 \\
\hline sa & 0,046 & 0,651 & 0,093 & 0,348 & 0,090 & 0,360 & 0,115 & 0,249 \\
\hline Intaille & 0,039 & 0,107 & 0,038 & 0,123 & 0,038 & 0,122 & 0,040 & $0,010^{*}$ \\
\hline pbv & $-0,040$ & 0,142 & & & & & & \\
\hline roe & & & 0,005 & 0,949 & & & & \\
\hline ne1 & & & & & $-0,060$ & 0,720 & & \\
\hline ne2 & & & & & & & $-0,002$ & 0,162 \\
\hline Const. & $-0,371$ & 0,136 & $-0,493$ & $0,039 * *$ & $-0,480$ & $0,046^{* *}$ & $-0,505$ & $0,032 * *$ \\
\hline Adjusted $\mathrm{R}^{2}$ & 0,04 & & 0,02 & & 0,02 & & 0,04 & \\
\hline Nb.Observations & 139 & & 139 & & 139 & & 139 & \\
\hline
\end{tabular}

Note. ${ }^{* * *}, * *$ and $*$ denote significance at $1 \%, 5 \%$ and $10 \%$.

Table 11. Bad news determinants during crisis period: results of CAR2 regression on rating characteristics with introduction of shareholding structure (institutional and foreign investors' presence)

\begin{tabular}{|c|c|c|c|c|c|c|c|c|}
\hline \multirow[b]{2}{*}{ Variables } & \multicolumn{2}{|c|}{ Model VII } & \multicolumn{2}{|c|}{ Model VIII } & \multicolumn{2}{|c|}{ Model IX } & \multicolumn{2}{|c|}{ Model X } \\
\hline & Coef. & $P-V a l$ & Coef. & $P$-Val & Coef. & $P$-Val & Coef. & $P$-Val \\
\hline ant & $-0,112$ & 0,150 & $-0,109$ & 0,161 & $-0,086$ & 0,287 & $-0,112$ & 0,152 \\
\hline $\operatorname{nin}$ & 0,069 & 0,441 & 0,072 & 0,423 & 0,062 & 0,480 & 0,074 & 0,400 \\
\hline $\mathrm{cc} 1$ & $-0,130$ & 0,340 & $-0,132$ & 0,333 & $-0,132$ & 0,331 & $-0,132$ & 0,333 \\
\hline amp & 0,039 & 0,327 & 0,038 & 0,334 & 0,039 & 0,320 & 0,038 & 0,335 \\
\hline $\mathrm{dn}$ & 0,008 & 0,905 & 0,011 & 0,876 & 0,020 & 0,786 & 0,010 & 0,882 \\
\hline sa & 0,090 & 0,368 & 0,092 & 0,353 & 0,097 & 0,325 & 0,092 & 0,350 \\
\hline Intaille & 0,038 & 0,116 & 0,039 & 0,120 & 0,039 & 0,106 & 0,037 & 0,132 \\
\hline ii & $-0,040$ & 0,813 & & & & & & \\
\hline nbre_ii & & & $-0,059$ & 0,893 & & & & \\
\hline ie & & & & & $-0,171$ & 0,298 & & \\
\hline nbre_ie & & & & & & & 0,016 & 0,932 \\
\hline Const. & $-0,458$ & 0,103 & $-0,447$ & 0,294 & $-0,486$ & $0,040 * *$ & $-0,494$ & $0,037 * *$ \\
\hline Adjusted $\mathrm{R}^{2}$ & 0,02 & & 0,02 & & 0,03 & & 0,02 & \\
\hline Nb.Observations & 139 & & 139 & & 139 & & 139 & \\
\hline
\end{tabular}

Note. ${ }^{* * *}, * *$ and $*$ denote significance at $1 \%, 5 \%$ and $10 \%$.

With respect to doubly rated firms, we firstly focus exclusively on first bad announcements from one agency. Then, we consider only the second bad announcements following these first ones.

Table 12. Determinants of first bad announcements during crisis period: Results of CAR1 regression on rating characteristics with introduction of firm business and firm size

\begin{tabular}{lllll}
\hline & \multicolumn{3}{l}{ Modèle I } & \multicolumn{3}{l}{ Modèle II } \\
\cline { 2 - 5 } Variables & Coef. & P-Val & Coef. & P-Val \\
\hline ant & 0,078 & $0,019^{* *}$ & $-0,162$ & $0,034^{* *}$ \\
nin & $-0,080$ & 0,298 & 0,030 & 0,714 \\
cc1 & $-0,147$ & 0,276 & $-0,124$ & 0,348 \\
amp & 0,004 & 0,919 & 0,008 & 0,831 \\
dn & 0,096 & 0,189 & 0,086 & 0,239 \\
sa & & & 0,071 & 0,461 \\
Intaille & & & 0,048 & $0,044^{* *}$ \\
\hline
\end{tabular}




\begin{tabular}{lllll}
\hline Const. & $-0,133$ & $0,075^{*}$ & $-0,665$ & $0,004^{* * *}$ \\
Adjusted R2 & 0,05 & & 0,10 & \\
Nb.Observations & 117 & & 117 & \\
\hline
\end{tabular}

Note. $* * *, * *$ and $*$ denote significance at $1 \%, 5 \%$ and $10 \%$

Table 13. Determinants of first bad announcements during crisis period: results of CAR1 regression on rating characteristics with introduction of financial performance and indebtedness

\begin{tabular}{|c|c|c|c|c|c|c|c|c|}
\hline \multirow[b]{2}{*}{ Variables } & \multicolumn{2}{|c|}{ Modèle III } & \multicolumn{2}{|c|}{ Modèle IV } & \multicolumn{2}{|c|}{ Modèle V } & \multicolumn{2}{|c|}{ Modèle VI } \\
\hline & Coef. & P-Val & Coef. & P-Val & Coef. & P-Val & Coef. & P-Val \\
\hline ant & $-0,176$ & $0,023 * *$ & $-0,168$ & $0,032 * *$ & $-0,157$ & $0,041 * *$ & $-0,167$ & $0,029 * *$ \\
\hline $\operatorname{nin}$ & 0,005 & 0,953 & 0,027 & 0,750 & 0,040 & 0,634 & 0,034 & 0,680 \\
\hline $\mathrm{ccl}$ & $-0,143$ & 0,285 & $-0,124$ & 0,351 & $-0,111$ & 0,405 & $-0,113$ & 0,395 \\
\hline amp & 0,013 & 0,730 & 0,009 & 0,816 & 0,012 & 0,754 & 0,008 & 0,821 \\
\hline $\mathrm{dn}$ & 0,078 & 0,283 & 0,087 & 0,232 & 0,092 & 0,209 & 0,080 & 0,272 \\
\hline $\mathrm{sa}$ & 0,042 & 0,675 & 0,068 & 0,478 & 0,064 & 0,502 & 0,086 & 0,376 \\
\hline Intaille & 0,049 & $0,042 * *$ & 0,050 & $0,042 * *$ & 0,048 & $0,048 * *$ & 0,049 & $0,040 * *$ \\
\hline pbv & $-0,029$ & 0,311 & & & & & & \\
\hline roe & & & $-0,032$ & 0,692 & & & & \\
\hline ne1 & & & & & $-0,108$ & 0,492 & & \\
\hline ne2 & & & & & & & $-0,002$ & 0,291 \\
\hline Const. & $-0,583$ & $0,016^{* *}$ & $-0,676$ & $0,004 * * *$ & $-0,640$ & $0,006^{* * *}$ & $-0,672$ & $0,003 * * *$ \\
\hline Adjusted $\mathrm{R}^{2}$ & 0,10 & & 0,09 & & 0,09 & & 0,10 & \\
\hline Nb.Observations & 117 & & 117 & & 117 & & 117 & \\
\hline
\end{tabular}

Note. $* * *, * *$ and $*$ denote significance at $1 \%, 5 \%$ and $10 \%$.

Table 14. Determinants of first bad announcements during crisis period: results of CAR1 regression on rating characteristics with introduction of shareholding structure (institutional and foreign investors')

\begin{tabular}{|c|c|c|c|c|c|c|c|c|}
\hline \multirow[b]{2}{*}{ Variables } & \multicolumn{2}{|c|}{ Modèle VII } & \multicolumn{2}{|c|}{ Modèle VIII } & \multicolumn{2}{|c|}{ Modèle IX } & \multicolumn{2}{|c|}{ Modèle X } \\
\hline & Coef. & $P-V a l$ & Coef. & $P-V a l$ & Coef. & $P-$ Val & Coef. & $P$-Val \\
\hline ant & $-0,166$ & $0,030 * *$ & $-0,150$ & $0,051^{*}$ & $-0,131$ & $0,093^{*}$ & $-0,159$ & $0,037 * *$ \\
\hline $\operatorname{nin}$ & 0,006 & 0,942 & 0,011 & 0,896 & 0,017 & 0,836 & 0,032 & 0,703 \\
\hline $\mathrm{cc} 1$ & $-0,109$ & 0,408 & $-0,124$ & 0,345 & $-0,126$ & 0,337 & $-0,133$ & 0,314 \\
\hline amp & 0,011 & 0,771 & 0,008 & 0,837 & 0,007 & 0,853 & 0,004 & 0,914 \\
\hline $\mathrm{dn}$ & 0,079 & 0,277 & 0,094 & 0,197 & 0,098 & 0,177 & 0,088 & 0,227 \\
\hline sa & 0,054 & 0,576 & 0,065 & 0,495 & 0,076 & 0,423 & 0,072 & 0,453 \\
\hline Intaille & 0,049 & $0,041 * *$ & 0,056 & $0,023 * *$ & 0,051 & $0,032 * *$ & 0,056 & $0,027 * *$ \\
\hline ii & $-0,184$ & 0,232 & & & & & & \\
\hline nbre_ii & & & $-0,544$ & 0,171 & & & & \\
\hline ie & & & & & $-0,244$ & 0,109 & & \\
\hline nbre_ie & & & & & & & $-0,200$ & 0,300 \\
\hline Const, & $-0,499$ & $0,061^{*}$ & $-0,236$ & 0,538 & $-0,663$ & $0,004 * * *$ & $-0,687$ & $0,003 * * *$ \\
\hline Adjusted $\mathrm{R}^{2}$ & 0,10 & & 0,10 & & 0,11 & & 0,10 & \\
\hline Nb.Observations & 117 & & 117 & & 117 & & 117 & \\
\hline
\end{tabular}

Note. $* * *, * *$ and $*$ denote significance at $1 \%, 5 \%$ and $10 \%$.

Table 15. Determinants of second bad announcements during crisis period: results of CAR1 regression on rating characteristics with introduction of firm business and firm size

\begin{tabular}{lllll}
\hline & Modèle I & \multicolumn{3}{c}{ Modèle II } \\
\cline { 2 - 5 } Variables & Coef. & $P$-Val & Coef. & $P$-Val \\
\hline ant & $-0,113$ & 0,140 & $-0,109$ & 0,168 \\
nin & $-0,077$ & 0,307 & $-0,015$ & 0,859 \\
cc1 & $-0,203$ & 0,124 & $-0,186$ & 0,160 \\
amp & 0,069 & $0,083^{*}$ & 0,069 & $0,084^{*}$ \\
\hline
\end{tabular}




\begin{tabular}{lllll}
\hline $\mathrm{dn}$ & $-0,054$ & 0,468 & $-0,060$ & 0,433 \\
sa & & & 0,031 & 0,748 \\
Intaille & & & 0,026 & 0,281 \\
Const. & $-0,053$ & 0,507 & $-0,342$ & 0,150 \\
Adjusted R & 0,03 & & 0,03 & \\
Nb.Observations & 117 & & 117 & \\
\hline
\end{tabular}

Note. $* * *, * *$ and $*$ denote significance at $1 \%, 5 \%$ and $10 \%$.

Table 16. Determinants of first bad announcements during crisis period: results of CAR1 regression on rating characteristics with introduction of financial performance and indebtedness

\begin{tabular}{|c|c|c|c|c|c|c|c|c|}
\hline \multirow[b]{2}{*}{ Variables } & \multicolumn{2}{|c|}{ Modèle III } & \multicolumn{2}{|c|}{ Modèle IV } & \multicolumn{2}{|c|}{ Modèle V } & \multicolumn{2}{|c|}{ Modèle VI } \\
\hline & Coef. & P-Val & Coef. & P-Val & Coef. & P-Val & Coef. & $P-V a l$ \\
\hline ant & $-0,122$ & 0,130 & $-0,099$ & 0,222 & $-0,108$ & 0,176 & $-0,115$ & 0,149 \\
\hline $\operatorname{nin}$ & $-0,040$ & 0,656 & $-0,009$ & 0,910 & $-0,012$ & 0,887 & $-0,011$ & 0,898 \\
\hline $\mathrm{cc} 1$ & $-0,205$ & 0,127 & $-0,186$ & 0,162 & $-0,184$ & 0,169 & $-0,175$ & 0,186 \\
\hline amp & 0,073 & $0,071^{*}$ & 0,067 & $0,098 *$ & 0,070 & $0,084^{*}$ & 0,069 & $0,083^{*}$ \\
\hline $\mathrm{dn}$ & $-0,066$ & 0,389 & $-0,063$ & 0,408 & $-0,057$ & 0,459 & $-0,065$ & 0,392 \\
\hline $\mathrm{sa}$ & 0,004 & 0,962 & 0,034 & 0,724 & 0,029 & 0,761 & 0,048 & 0,622 \\
\hline Intaille & 0,026 & 0,280 & 0,024 & 0,324 & 0,026 & 0,288 & 0,028 & 0,259 \\
\hline pbv & $-0,027$ & 0,350 & & & & & & \\
\hline roe & & & 0,048 & 0,565 & & & & \\
\hline ne1 & & & & & $-0,028$ & 0,860 & & \\
\hline ne2 & & & & & & & $-0,002$ & 0,253 \\
\hline Const, & $-0,260$ & 0,303 & $-0,324$ & 0,178 & $-0,335$ & 0,165 & $-0,350$ & 0,140 \\
\hline Adjusted $\mathrm{R}^{2}$ & 0,03 & & 0,02 & & 0,02 & & 0,03 & \\
\hline Nb.Observations & 117 & & 117 & & 117 & & 117 & \\
\hline
\end{tabular}

Note. $* * *, * *$ and $*$ denote significance at $1 \%, 5 \%$ and $10 \%$.

Table 17. Determinants of first bad announcements during crisis period: results of CAR1 regression on rating characteristics with introduction of shareholding structure (institutional and foreign investors' presence)

\begin{tabular}{|c|c|c|c|c|c|c|c|c|}
\hline \multirow[b]{2}{*}{ Variables } & \multicolumn{2}{|c|}{ Modèle VII } & \multicolumn{2}{|c|}{ Modèle VIII } & \multicolumn{2}{|c|}{ Modèle IX } & \multicolumn{2}{|c|}{ Modèle X } \\
\hline & Coef. & $P-V a l$ & Coef. & $P-V a l$ & Coef. & $P-V a l$ & Coef. & $P-V a l$ \\
\hline ant & $-0,112$ & 0,161 & $-0,106$ & 0,187 & $-0,099$ & 0,230 & $-0,111$ & 0,163 \\
\hline $\operatorname{nin}$ & $-0,026$ & 0,765 & $-0,020$ & 0,816 & $-0,019$ & 0,820 & $-0,015$ & 0,854 \\
\hline $\operatorname{cc} 1$ & $-0,182$ & 0,171 & $-0,186$ & 0,163 & $-0,185$ & 0,165 & $-0,190$ & 0,153 \\
\hline $\mathrm{amp}$ & 0,071 & $0,078 *$ & 0,069 & $0,085^{*}$ & 0,068 & $0,089 *$ & 0,070 & $0,080^{*}$ \\
\hline $\mathrm{dn}$ & $-0,062$ & 0,417 & $-0,057$ & 0,453 & $-0,056$ & 0,462 & $-0,061$ & 0,426 \\
\hline sa & 0,024 & 0,807 & 0,030 & 0,760 & 0,033 & 0,735 & 0,031 & 0,745 \\
\hline lntaille & 0,027 & 0,278 & 0,029 & 0,260 & 0,027 & 0,268 & 0,020 & 0,436 \\
\hline ii & $-0,092$ & 0,559 & & & & & & \\
\hline nbre_ii & & & $-0,148$ & 0,717 & & & & \\
\hline ie & & & & & $-0,077$ & 0,624 & & \\
\hline nbre_ie & & & & & & & 0,161 & 0,413 \\
\hline Const. & $-0,259$ & 0,348 & $-0,225$ & 0,574 & $-0,340$ & 0,154 & $-0,319$ & 0,183 \\
\hline Adjusted R2 & 0,02 & & 0,02 & & 0,02 & & 0,03 & \\
\hline Nb.Observations & 117 & & 117 & & 117 & & 117 & \\
\hline
\end{tabular}

Note. ${ }^{* * *}, * *$ and $*$ denote significance at $1 \%, 5 \%$ and $10 \%$.

Results reveal that during crisis period expected bad rating announcements of big firms and with considerable magnitude have great impact on stock prices. Conversely, market reaction to downgrades is independent of firm financial performance, indebtedness level and shareholding structure. These findings point out once again the crisis faith towards rating agencies. First, expected announcements have stronger impact on stock prices than surprise ones. Investors respond to well thought bad announcements on what their investment decisions were 
based more than they do to sudden announcements that only source is rating agencies. Thus, expected rating announcements do not guide investors on investment decisions but seem to strengthen their positions (Norden \& Weber, 2004; Di Cesare, 2006). Second, magnitude is significant for repeated downgrades. This result denotes that investors are more sensitive to important successive downgrades during crisis period than slight ones. In fact, downgrade magnitude is a warning sign of firm financial health. Therefore, it seems logical that investors intensively react to downgrades with important magnitudes. The latter determinant of downgrades' impact on stock prices is the firm size. Downgrades have great impact on stock prices of big firms. Indeed, their financial difficulties lead to accentuate the financial market turmoil and to cause huge losses for investors.

\subsection{Good News}

The correlation matrix (Note 4) combined with independence Chi square test and Spearman correlation coefficient reveal that during crisis the factors explaining good rating announcements effects on stock prices are the initial rating level, the magnitude, the double rating and the firm sector.

Table 18. Correlation matrix of endogenous and exogenous variables relative to good rating news during crisis period

\begin{tabular}{|c|c|c|c|c|c|c|c|c|c|c|c|c|c|c|c|c|c|c|}
\hline & carl & car2 & ant & nin & $c c 1$ & $c c 2$ & $a m p$ & $d n$ & $S a$ & $p b v$ & $i i$ & nbre_ii & $i e$ & nbre-ie & nel & $n e 2$ & Intaille & roe \\
\hline carl & 1 & 0.948 & 0.154 & -0.011 & 0.095 & 0.066 & 0.655 & 0.381 & 0.560 & -0.429 & -0.715 & -0.734 & -0.006 & -0.050 & 0.557 & 0.482 & 0.482 & 0.309 \\
\hline car2 & & 1 & 0.315 & 0.043 & 0.242 & 0.085 & 0.834 & 0.321 & 0.589 & -0.397 & -0.770 & -0.887 & -0.049 & -0.154 & 0.073 & 0.585 & 0.673 & 0.476 \\
\hline Ant & & & 1 & 0.025 & 0.189 & 0.244 & 0.318 & 0.304 & -0.026 & 0.350 & -0.250 & -0.360 & -0.207 & -0.317 & -0.177 & 0.047 & 0.384 & 0.671 \\
\hline Nin & & & & 1 & 0.330 & 0.337 & 0.082 & 0.284 & -0.152 & 0.261 & 0.064 & -0.153 & -0.531 & 0.079 & -0.419 & 0.158 & -0.246 & 0.237 \\
\hline$c c 1$ & & & & & 1 & 0.471 & 0.477 & -0.194 & 0.055 & -0.102 & -0.496 & -0.450 & -0.176 & -0.293 & -0.232 & 0.029 & 0.242 & 0.393 \\
\hline$c c 2$ & & & & & & 1 & 0.116 & 0.122 & -0.337 & 0.180 & -0.387 & -0.264 & -0.372 & -0.556 & 0.295 & -0.216 & -0.205 & 0.473 \\
\hline$A m p$ & & & & & & & 1 & 0.064 & 0.553 & -0.260 & -0.791 & -0.957 & -0.043 & -0.256 & -0.096 & 0.523 & 0.778 & 0.579 \\
\hline$D n$ & & & & & & & & 1 & -0.284 & 0.290 & 0.264 & -0.146 & -0.679 & 0.003 & 0.222 & 0.150 & -0.172 & 0.122 \\
\hline$S a$ & & & & & & & & & 1 & -0.387 & -0.282 & -0.459 & 0.531 & 0.358 & -0.255 & 0.805 & 0.730 & 0.096 \\
\hline$P b v$ & & & & & & & & & & 1 & 0.249 & 0.265 & -0.194 & -0.110 & -0.095 & -0.200 & -0.339 & 0.235 \\
\hline$i i$ & & & & & & & & & & & 1 & 0.832 & 0.185 & 0.402 & -0.418 & -0.316 & -0.436 & -0.381 \\
\hline nbre_ii & & & & & & & & & & & & 1 & 0.119 & 0.384 & -0.036 & -0.449 & -0.684 & -0.619 \\
\hline$i e$ & & & & & & & & & & & & & 1 & -0.007 & -0.157 & -0.029 & 0.343 & -0.049 \\
\hline nbre-ie & & & & & & & & & & & & & & 1 & -0.255 & 0.568 & -0.099 & -0.506 \\
\hline nel & & & & & & & & & & & & & & & 1 & -0.182 & -0.355 & -0.276 \\
\hline$n e 2$ & & & & & & & & & & & & & & & & 1 & 0.525 & 0.028 \\
\hline Intaille & & & & & & & & & & & & & & & & & 1 & 0.494 \\
\hline roe & & & & & & & & & & & & & & & & & & 1 \\
\hline
\end{tabular}

Note. CAR (1 2) cumulative abnormal return one day before the announcement, relative to stock adjusted model (1) or market adjusted model (2); ant: anticipation criterion, nin: rating initial level (speculative or investment grade); cc (1): rating change class (from speculative to investment grade); amp: change amplitude; dn: double rating; sa: business firm (financial and banking or not); pbv: price book value; ii: institutional investors' presence in shareholding in terms of monetary values; nbre_ii: institutional investors' presence in shareholding in terms of shareholders number; ie: foreign investors' presence in shareholding in terms of monetary values; nbre_ie: foreign investors' presence in shareholding in terms of shareholders number; ne1: total debt / total assets; ne2: total debt / equity; lntaille: natural logarithm of total assets; roe: return on equity (Net income /equity).

Table 19. Results of Chi square test and Spearman coefficient

\begin{tabular}{lcccc}
\hline & Khi-carré $\left(\right.$ CAR $\left._{l}\right)$ & p-value & Khi-carré $\left(\right.$ CAR $\left._{2}\right)$ & $p$-value \\
\hline ant & 1,143 & 0,285 & 1,143 & 0,285 \\
nin & $4,571^{* *}$ & 0,033 & $4,571^{* *}$ & 0,033 \\
$c c 1$ & 2,571 & 0,109 & 2,571 & 0,109 \\
$c c 2$ & 1,143 & 0,285 & 1,143 & 0,285 \\
amp & $13^{* * *}$ & 0,002 & $13^{* * *}$ & 0,002 \\
$d n$ & $7,143^{* * *}$ & 0,008 & $7,143^{* * *}$ & 0,008 \\
sa & $4,571^{* *}$ & 0,033 & $4,571^{* *}$ & 0,033 \\
Variable & $\rho\left(C A R_{l}\right)$ & $p$-value & $\rho s\left(C A R_{2}\right)$ & $p$-value \\
lntaille & $-0,054$ & 0,852 & 0,002 & 0,994 \\
\hline
\end{tabular}




\begin{tabular}{lllll}
\hline$p b v$ & $-0,389$ & 0,169 & $-0,353$ & 0,214 \\
roe & 0,068 & 0,817 & 0,112 & 0,702 \\
$n e 1$ & 0,389 & 0,169 & 0,345 & 0,226 \\
$n e 2$ & 0,380 & 0,179 & 0,287 & 0,318 \\
$i i$ & $-0,279$ & 0,333 & $-0,371$ & 0,191 \\
$n b r e \_i i$ & $-0,309$ & 0,280 & $-0,336$ & 0,239 \\
ie & $-0,050$ & 0,863 & 0,024 & 0,934 \\
Nbre_ie & $-0,264$ & 0,361 & $-0,235$ & 0,417 \\
\hline
\end{tabular}

ant: anticipation criterion, nin: rating initial level (speculative or investment grade); sa: business firm (financial and banking or not); pbv: price book value; ii : institutional investors' presence in shareholding in terms of monetary values; nbre_ii : institutional investors' presence in shareholding in terms of shareholders number; ie: foreign investors' presence in shareholding in terms of monetary values; nbre ie: foreign investors' presence in shareholding in terms of shareholders number; ne1: total debt / total assets; ne2: total debt / equity; lntaille: natural logarithm of total assets; roe: return on equity (Net income /equity).

First, it seems that investors take into account upgrades of speculative equities more than they do of investment grade ones (Hand et al., 1992; Jorion et Zangh, 2007). In the former case, the probability of default is very important and highly likely. Furthermore, the significance of double rating factor corroborates the theoretical finding of Raimbourg (1990). Double rating permits to alleviate interest conflicts between firms and rating agencies. These could perhaps be complacent in rating firms due to paid fees. That's why; multiple ratings allow thwarting this problem. This finding joins those of Hand et al. (1992) and highlights once again the important effect of double or multiple notations on investors. Otherwise, the upgrade magnitude indicates the improvement of firm's repayment ability, and thus the firm's financial soundness. Therefore, big upgrades have more impact on stock prices than small ones. Finally, as regards firm's characteristics, it seems that only firm sector and institutional presence in shareholding explain market reaction to upgrades during crisis period. Indeed, financial securities upgrades have higher repercussion on stock prices than non-financial ones. This can be explained by the banking and financial origin of 2008 crisis. Also, the correlation matrix reveals significant relation between institutional presence in shareholding and upgrades' impact on stock prices. This is explained by investment constraints imposed on institutional investors that are required to sell speculative securities and to buy investment grade ones. Consequently, they react to upgrades and generate a significant increase on stock prices (Halek \& Eckles, 2010).

\subsection{Neutral News or Assertions}

The Variance Inflation Factors allows to invalid presence of multicollinearity between independent variables. Besides, the correlation matrix (Note 5) eliminates presence of significant variables correlations.

Table 20. VIF test results: explanatory variables of market reaction to neutral rating news during crisis period

\begin{tabular}{lcccccccccccccc}
\hline Variables & ant & nin & $d n$ & $s a$ & $p b v$ & ii & nbre_ii & ie & nbre_ie & nel & ne2 & lntaille & roe & Mean VIF \\
\hline VIF & 1.26 & 1.96 & 1.36 & 2.13 & 1.22 & 1.75 & 1.85 & 2.06 & 2.39 & 1.97 & 1.84 & 2.74 & 1.09 & 1.82 \\
\hline
\end{tabular}

Note. ant: anticipation criterion, nin: rating initial level (speculative or investment grade); sa: business firm (financial and banking or not); pbv: price book value; ii : institutional investors' presence in shareholding in terms of monetary values; nbre ii : institutional investors' presence in shareholding in terms of shareholders number; ie: foreign investors' presence in shareholding in terms of monetary values; nbre_ie: foreign investors' presence in shareholding in terms of shareholders number; ne1: total debt / total assets; ne2: total debt / equity; Intaille: natural logarithm of total assets; roe: return on equity (Net income /equity).

In model I, we regress the cumulative abnormal return $\mathrm{CAR}_{1}$ on structural variables, such as anticipation, initial level of rating and double notation. After, we gradually introduce control variables, such as business and size firm (Model II), financial performance (Models III and IV), indebtedness (Models V and VI), and shareholding structure: institutional investors (Models VII and VIII) and foreign investors (Models IX and X). 
Table 21. Correlation matrix of endogenous and exogenous variables relative to neutral rating news during crisis period

\begin{tabular}{|c|c|c|c|c|c|c|c|c|c|c|c|c|c|c|c|}
\hline & carl & car2 & ant & $\operatorname{nin}$ & $d n$ & $S a$ & $p b v$ & $i i$ & nbre_ii & ie & nbre-ie & nel & $n e 2$ & Intaille & roe \\
\hline carl & 1 & 0.946 & -0.481 & 0.036 & -0.100 & 0.120 & 0.165 & 0.251 & 0.066 & 0.021 & 0.081 & 0.009 & 0.031 & 0.011 & 0.053 \\
\hline car2 & & 1 & -0.320 & 0.062 & -0.085 & 0.127 & 0.166 & 0.302 & 0.111 & 0.076 & 0.128 & 0.041 & 0.104 & 0.052 & -0.023 \\
\hline Ant & & & 1 & 0.161 & 0.068 & -0.294 & -0.077 & -0.078 & -0.014 & -0.056 & -0.033 & 0.202 & -0.099 & -0.234 & -0.125 \\
\hline Nin & & & & 1 & 0.304 & -0.417 & -0.016 & -0.086 & -0.239 & -0.071 & -0.066 & 0.447 & -0.151 & -0.515 & 0.098 \\
\hline$D n$ & & & & & 1 & -0.293 & -0.016 & 0.014 & 0.010 & 0.221 & 0.168 & 0.180 & -0.005 & -0.001 & -0.061 \\
\hline$S a$ & & & & & & 1 & -0.199 & -0.050 & 0.178 & 0.197 & 0.231 & -0.436 & 0.414 & 0.521 & -0.078 \\
\hline$P b v$ & & & & & & & 1 & 0.038 & -0.011 & -0.027 & -0.096 & 0.224 & 0.051 & -0.137 & -0.099 \\
\hline$i i$ & & & & & & & & 1 & 0.558 & 0.156 & 0.016 & 0.144 & 0.052 & 0.135 & -0.116 \\
\hline nbre_ii & & & & & & & & & 1 & 0.144 & 0.244 & -0.016 & 0.142 & 0.358 & -0.095 \\
\hline ie & & & & & & & & & & 1 & 0.658 & -0.295 & 0.115 & 0.260 & -0.031 \\
\hline nbre-ie & & & & & & & & & & & 1 & -0.357 & 0.235 & 0.403 & -0.047 \\
\hline nel & & & & & & & & & & & & 1 & 0.040 & -0.314 & 0.069 \\
\hline$n e 2$ & & & & & & & & & & & & & 1 & 0.549 & -0.103 \\
\hline lntaille & & & & & & & & & & & & & & 1 & -0.086 \\
\hline roe & & & & & & & & & & & & & & & 1 \\
\hline
\end{tabular}

Note. CAR (1 2) cumulative abnormal return one day before the announcement, relative to stock adjusted model (1) or market adjusted model (2); ant: anticipation criterion, nin: rating initial level (speculative or investment grade); dn: double rating; sa: business firm (financial and banking or not); pbv: price book value; ii: institutional investors' presence in shareholding in terms of monetary values; nbre_ii: institutional investors' presence in shareholding in terms of shareholders number; ie: foreign investors' presence in shareholding in terms of monetary values; nbre_ie: foreign investors' presence in shareholding in terms of shareholders number; ne1: total debt / total assets; ne2: total debt / equity; lntaille: natural logarithm of total assets; roe: return on equity (Net income /equity).

Table 22. Neutral news determinants during crisis period: results of CAR1 regression on rating characteristics with introduction of firm business and firm size

\begin{tabular}{lllll}
\hline & Model I & \multicolumn{3}{l}{ Model II } \\
\cline { 2 - 5 } Variables & Coef. & $P$-Val & Coef. & $P$-Val \\
\hline ant & -0.269 & $0.000^{* * *}$ & -0.270 & $0.000^{* * *}$ \\
nin & 0.758 & 0.319 & 0.066 & 0.450 \\
dn & -0.066 & 0.528 & -0.058 & 0.615 \\
sa & & & 0.134 & 0.869 \\
Intaille & & & -0.005 & 0.718 \\
Const. & 0.070 & 0.451 & 0.114 & 0.600 \\
Adjusted $\mathrm{R}^{2}$ & 0.2571 & & 0.2586 & \\
Nb.Observations & 52 & & 52 & \\
\hline
\end{tabular}

Note. $* * *, * *$ and $*$ denote significance at $1 \%, 5 \%$ and $10 \%$.

Table 23. Neutral news determinants during crisis period: results of CAR1 regression on rating characteristics with introduction of financial performance and indebtedness

\begin{tabular}{lllllllll}
\hline & Model III & \multicolumn{3}{c}{ Model IV } & Model V & \multicolumn{3}{c}{ Model VI } \\
\cline { 2 - 9 } Variables & Coef. & $P$-Val & Coef. & $P$-Val & Coef. & $P$-Val & Coef. & $P$-Val \\
\hline Ant & -0.259 & $0.001^{* * *}$ & -0.271 & $0.001^{* * *}$ & -0.273 & $0.000^{* * *}$ & -0.271 & $0.001^{* * *}$ \\
Nin & 0.073 & 0.403 & 0.066 & 0.413 & 0.051 & 0.521 & 0.062 & 0.451 \\
dn & -0.054 & 0.633 & -0.057 & 0.500 & -0.057 & 0.613 & -0.058 & 0.498 \\
Sa & 0.030 & 0.717 & 0.012 & 0.877 & 0.026 & 0.771 & 0.009 & 0.908 \\
Lntaille & -0.004 & 0.793 & -0.005 & 0.760 & -0.005 & 0.718 & -0.007 & 0.727 \\
Pbv & 0.020 & 0.220 & & & & & & \\
Roe & & & -0.007 & 0.884 & & & & \\
ne1 & & & & & 0.117 & 0.620 & & 0.013 \\
ne2 & & & & & & & 0.854 \\
Const. & 0.042 & 0.855 & 0.116 & 0.520 & 0.088 & 0.719 & 0.127 & 0.511 \\
\hline
\end{tabular}




\begin{tabular}{lllll}
\hline Adjusted $\mathrm{R}^{2}$ & 0.2747 & 0.2589 & 0.2644 & 0.1604 \\
Nb.Observations & 52 & 52 & 52 & 52 \\
\hline
\end{tabular}

Note. $* * *, * *$ and $*$ denote significance at $1 \%, 5 \%$ and $10 \%$.

Table 24. Neutral news determinants during crisis period: results of CAR1 regression on rating characteristics with introduction of shareholding structure (institutional and foreign investors' presence)

\begin{tabular}{|c|c|c|c|c|c|c|c|c|}
\hline \multirow[b]{2}{*}{ Variables } & \multicolumn{2}{|c|}{ Model VII } & \multicolumn{2}{|c|}{ Model VIII } & \multicolumn{2}{|c|}{ Model IX } & \multicolumn{2}{|c|}{ Model X } \\
\hline & Coef. & $P-V a l$ & Coef. & $P-V a l$ & Coef. & $P-V a l$ & Coef. & P-Val \\
\hline ant & -0.259 & $0.000 * * *$ & -0.275 & $0.000 * * *$ & -0.270 & $0.001 * * *$ & -0.362 & $0.000 * * *$ \\
\hline $\operatorname{nin}$ & 0.072 & 0.434 & 0.071 & 0.379 & 0.065 & 0.417 & 0.114 & 0.179 \\
\hline $\mathrm{dn}$ & -0.054 & 0.633 & -0.058 & 0.489 & -0.064 & 0.467 & -0.072 & 0.413 \\
\hline sa & 0.038 & 0.640 & 0.013 & 0.867 & 0.009 & 0.911 & -0.104 & 0.232 \\
\hline Intaille & -0.010 & 0.563 & -0.010 & 0.604 & -0.006 & 0.740 & 0.003 & 0.870 \\
\hline ii & 0.218 & $0.058 *$ & & & & & & \\
\hline nbre_ii & & & 0.214 & 0.386 & & & & \\
\hline ie & & & & & 0.059 & 0.784 & & \\
\hline nbre_ie & & & & & & & 0.161 & 0.432 \\
\hline Const. & -0.022 & 0.923 & -0.037 & 0.881 & 0.065 & 0.726 & 0.121 & 0.505 \\
\hline Adjusted $\mathrm{R}^{2}$ & 0.3157 & & 0.1738 & & 0.1611 & & 0.2812 & \\
\hline Nb.Observations & 52 & & 52 & & 52 & & 52 & \\
\hline
\end{tabular}

Note. $* * *, * *$ and $*$ denote significance at $1 \%, 5 \%$ and $10 \%$.

For results' robustness, we get back on regressions by considering $\mathrm{CAR}_{2}$ as the endogenous variable. We find same results.

Table 25. Neutral news determinants during crisis period: results of CAR2 regression on rating characteristics with introduction of firm business and firm size

\begin{tabular}{|c|c|c|c|c|}
\hline \multirow[b]{2}{*}{ Variables } & \multicolumn{2}{|l|}{ Model I } & \multicolumn{2}{|c|}{ Modele II } \\
\hline & Coef. & P-Val & Coef. & $P$-Val \\
\hline ant & -0.196 & $0.012 * *$ & -0.183 & $0.034 * *$ \\
\hline $\operatorname{nin}$ & 0.081 & 0.365 & 0.105 & 0.336 \\
\hline $\mathrm{dn}$ & -0.068 & 0.562 & -0.067 & 0.611 \\
\hline $\mathrm{sa}$ & & & 0.033 & 0.698 \\
\hline Intaille & & & 0.005 & 0.767 \\
\hline Const. & 0.056 & 0.590 & -0.024 & 0.922 \\
\hline Adjusted $\mathrm{R}^{2}$ & 0.1265 & & 0.2453 & \\
\hline Nb.Observations & 52 & & 52 & \\
\hline
\end{tabular}

Note. $* * * * *$ and $*$ denote significance at $1 \%, 5 \%$ and $10 \%$.

Table 26. Neutral news determinants during crisis period: results of CAR2 regression on rating characteristics with introduction of financial performance and indebtedness

\begin{tabular}{lllllllll}
\hline & Model III & \multicolumn{3}{c}{ Model IV } & \multicolumn{3}{c}{ Model V } & \multicolumn{2}{c}{ Model VI } \\
\cline { 2 - 8 } Variables & Coef. & P-Val & Coef. & P-Val & Coef. & P-Val & Coef. & $P$-Val \\
\hline ant & -0.168 & $0.053^{* *}$ & -0.190 & $0.032^{* *}$ & -0.188 & $0.034^{* *}$ & -0.186 & $0.033^{* *}$ \\
nin & 0.115 & 0.220 & 0.107 & 0.258 & 0.086 & 0.391 & 0.096 & 0.325 \\
dn & -0.062 & 0.528 & -0.065 & 0.512 & -0.066 & 0.607 & -0.066 & 0.504 \\
sa & 0.056 & 0.563 & 0.031 & 0.749 & 0.051 & 0.618 & 0.023 & 0.816 \\
lntaille & 0.007 & 0.727 & 0.005 & 0.818 & 0.005 & 0.787 & 0.000 & 0.989 \\
pbv & 0.029 & 0.238 & & & & & & \\
roe & & & -0.029 & 0.627 & & 0.154 & 0.617 & \\
ne1 & & & & & & & 0.003 \\
ne2 & & & & & & & 0.648 \\
\hline
\end{tabular}




\begin{tabular}{|c|c|c|c|c|c|c|c|c|}
\hline Const. & -0.124 & 0.579 & -0.016 & 0.938 & -0.058 & 0.837 & 0.013 & 0.952 \\
\hline Adjusted $\mathrm{R}^{2}$ & 0.0471 & & 0.0220 & & 0.1411 & & 0.0214 & \\
\hline Nb.Observations & 52 & & 52 & & 52 & & 52 & \\
\hline
\end{tabular}

Note. $* * * * *$ and $*$ denote significance at $1 \%, 5 \%$ and $10 \%$

Table 27. Bad news determinants during crisis period: results of CAR2 regression on rating characteristics with introduction of shareholding structure (institutional and foreign investors' presence)

\begin{tabular}{|c|c|c|c|c|c|c|c|c|}
\hline \multirow[b]{2}{*}{ Variables } & \multicolumn{2}{|c|}{ Model VII } & \multicolumn{2}{|c|}{ Model VIII } & \multicolumn{2}{|c|}{ Model IX } & \multicolumn{2}{|c|}{ Model X } \\
\hline & Coef. & P-Val & Coef. & P-Val & Coef. & P-Val & Coef. & $P-V a l$ \\
\hline ant & -0.169 & $0.024 * *$ & -0.190 & $0.029 * *$ & -0.185 & $0.034 * *$ & -0.190 & $0.029 * *$ \\
\hline $\operatorname{nin}$ & 0.114 & 0.327 & 0.112 & 0.236 & 0.105 & 0.268 & 0.093 & 0.388 \\
\hline dn & -0.062 & 0.630 & -0.067 & 0.493 & -0.081 & 0.428 & -0.082 & 0.549 \\
\hline $\mathrm{sa}$ & 0.068 & 0.433 & 0.034 & 0.725 & 0.024 & 0.807 & 0.022 & 0.807 \\
\hline Intaille & -0.001 & 0.944 & -0.000 & 0.996 & 0.004 & 0.857 & -0.001 & 0.934 \\
\hline ii & 0.296 & $0.031 * *$ & & & & & & \\
\hline nbre_ii & & & 0.278 & 0.335 & & & & \\
\hline ie & & & & & 0.139 & 0.581 & & \\
\hline nbre_ie & & & & & & & 0.217 & 0.350 \\
\hline Const. & -0.209 & 0.427 & -0.221 & 0.448 & 0.007 & 0.971 & 0.021 & 0.932 \\
\hline Adjusted $\mathrm{R}^{2}$ & 0.2227 & & 0.0372 & & 0.0235 & & 0.1494 & \\
\hline Nb.Observations & 52 & & 52 & & 52 & & 52 & \\
\hline
\end{tabular}

Note. $* * * * *$ and $*$ denote significance at $1 \%, 5 \%$ and $10 \%$.

Regression results denote that the anticipation of the announcement and the presence of institutional investors are the most important determinants of market reaction to assertions during crisis period. This finding is mainly explained by investors risk aversion especially during financial turmoil. Assertions reveal constancy and not improvement of firms' repayment ability. Therefore, they maintain priority position of bondholders to stockholders especially during crisis. This enhances investors' reject to affirmed securities and their prices' decrease. Negative investor's response to assertions increases when assertion is expected. Also, affirmations could have much greater impact when there is an important institutional presence in shareholding. Indeed, institutional investors have to take short positions on risky assets, especially during crisis period. That's why an assertion pushes them to sell affirmed assets, which generates prices decline. Furthermore, it seems that market reaction to assertions is stronger for speculative than investment grade securities.

\section{Conclusion}

This paper aims to explain market reaction to rating announcements during crisis period. Its main contribution to previous works is twofold. Firstly, it offers a new context of rating determinants study, which is the 2008 crisis period. Secondly, this paper presents three potential determinants of market reaction to rating announcements during crisis period: the announcement anticipation, the double notation and foreign investors' presence in shareholding. An event study allows measuring market reaction to rating announcements. Its proxy is the cumulative abnormal return during the period surrounding the announcement date. For bad and neutral news, we regress the cumulative abnormal returns on structural factors related to the rating. Then, we gradually introduce firms' characteristics as control variables, such as business, size, indebtedness, financial performance and shareholding structure: institutional and foreign investors' presence. For good news, due to the small sample size, we just establish correlation matrix and Chi square independence test to check significant relation between market reaction to upgrades and both of ratings and firms' characteristics. We find firstly the influence of anticipation, rating magnitude and firm size on market reactions to bad rating news. Otherwise, good rating announcements' effects on stock prices depend on initial rating level, change magnitude and firms' sector. In fact, financial and banking stocks have greater impact on markets than non-financial ones. Besides, institutional investors' presence in shareholding enhances the stock price increase following good and neutral news announcements. Assertions' effects are also dependent upon the announcements' anticipation. Globally, results point out investors' loss faith towards rating agencies during the 2008 financial crisis. These should re-launch a favourable image on financial markets. Accordingly, they have to revise their methodologies in ratings assignments and changes. At last, rating agencies have to be cautious in rating especially large firm because they are of great interest to investors. 


\section{References}

Avramov D., Chordia, T., Jostova, G., \& Philipov, A. (2009). Credit Ratings and the Cross Section of Stock Returns. Working Paper, EFA Athens Meeting Paper.

Azouz, D., Boudriga, A., \& Mamoghli, C. (2011). Does American Stock Market React differently to Rating Announcements During Crisis Period? The Case of the 2008 Worldwide Financial Crisis. Working Paper, Economic Research Forum, Egypt, 601.

Bonini S., Pettinato, O., \& Salvi, A. (2009). Do target prices predict rating changes? Working Paper, SSRN id 1364464.

Creighton, A., Gower, L., \& Richards, A. J. (2007). The impact of rating changes in Australian financial markets. Pacific-Basin Finance Journal, 15, 1-17. http://dx.doi.org/10.1016/j.pacfin.2006.04.003

Di Cesare, A. (2006). Do market based indicators anticipate rating agencies? Evidence for international Banks. Working Paper, Temi di discussione del servizio study, Banca d'Italia, 593.

Dichev, I. D., \& Piotroski, J. D. (2001). The long run stock returns following bond ratings changes. The Journal of Finance, 56(1), 173-203. http://dx.doi.org/10.1111/0022-1082.00322

Ederington, L. H., \& Goh, J. C. (1993). Is a bond rating downgrade bad news, good news or no news for stockholders? The Journal of 2001-2008. http://dx.doi.org/10.1111/j.1540-6261.1993.tb05139.x

Frankel, J. A., \& Schmukler, S. L. (1996). Country fund discounts and the Mexican crisis of 1994: Did Mexican residents turn pessimistic before international investors? Open Econ. Rev, 7, 511-534. http://dx.doi.org/10.1007/BF01886211

Frankel, J. A., \& Schmukler, S. L. (1998a). Country Funds and Asymmetric Information. World Bank Policy Research, Working Paper c1886. Retrieved from http://wbln0018.worldbank.org/research/workpapers.nsf/

Gropp, R., \& Richards, A. J. (2001). Rating Agencies Actions and the Pricing of Debt and Equity of European Banks: What Can We Infer about Private Sector Monitoring of Bank Soundness? Working Paper 76, European Central Bank.

Halek, M., \& Eckles, D. E. (2010). Effects of analyst's ratings on insurer stock returns: Evidence of asymmetric $\begin{array}{lllll}\text { responses. Journal of Insurance } & \text { 801-827. }\end{array}$ http://dx.doi.org/10.1111/j.1539-6975.2010.01368.x

Hand, J. R. M., Holthausen, R. W., \& Leftwich, R. W. (1992). The effect of bond rating agency announcements on bond and stock prices. The Journal of Finance, 47(2), 733-752. http://dx.doi.org/10.1111/j.1540-6261.1992.tb04407.x

Holthausen, R. W., \& Leftwich, R. W. (1986). The effect of bond rating changes on common stock prices. The Journal of Financial Economics, 17, 57-89. http://dx.doi.org/10.1016/0304-405X(86)90006-1

John, K, Ravid, S. A., \& Reisel, N. (2005). Senior and Subordinated Issues, Debt Ratings and Price Impact. Working Paper, SSRN id 670443.

Jorion, P., \& Zhang, G. (2007). Non-linear effects of bond ratings changes. Journal of Fixed Income, 16(4), 45-59. http://dx.doi.org/10.3905/jfi.2007.683317

Karolyi, G. A. (2002). Did the Asian Financial Market scare foreign investors out of Japan? Pacific Basin Financial Journal, 10, 411-442. http://dx.doi.org/10.1016/S0927-538X(02)00067-7

Linciano, N. (2008). The informational content of ratings, the effects of rating agencies actions on stock prices for the Italian Case. Working Paper, Commissione Nazionale per le Società e la Borsa (CONSOB), Division of Markets and Economic Studies via G.B Martini.

Micu, M., Remonola, E. M., \& Wooldridge, P. D. (2004). The price impact of rating announcements: Evidence from the credit default swap market. Bis Quaterly Review, 55-65.

Nayar, N., \& Rozeff, M. S. (1994). Ratings, Commercial Paper, and equity returns. The Journal of Finance, 49, 1431-1449. http://dx.doi.org/10.1111/j.1540-6261.1994.tb02460.x

Norden, L., \& Weber, M. (2004). Informational efficiency of credit default swap and stock markets: The impact of credit rating announcements. Journal of Banking and Finance, 28, 2813-2843. http://dx.doi.org/10.1016/j.jbankfin.2004.06.011 
Purda, L. D. (2006). Stock Market reaction to anticipated versus surprise rating changes. Working Paper, Queen's University.

Raimbourg, P. (1990). Les agences de rating. Editions Economica.

Schweitzer, R., Szewczyk, S., \& Varma, R. (1992). Bond Rating Agencies and their Role in Bank Market Discipline. Journal of Financial Services Research, 6, 249-264. http://dx.doi.org/10.1007/BF01047083

Sunder, L. S. (1991). The stock price effect of risky versus safe debt. The Journal of Financial and Quantitative Analysis, 26(4), 549-558. http://dx.doi.org/10.2307/2331411

\section{Notes}

Note 1. If two ratings occur during the same event window, the event is considered as a single one and the day 0 corresponds to the first announcement day.

Note 2. Please refer to table 5 in the annexes.

Note 3. CAR1 is the CAR calculated by the stock adjusted model, CAR2 is calculated by the market adjusted model.

Note 4. Please refer to table 18 in the annexes.

Note 5 . Please refer to table 21 in the annexes.

\section{Copyrights}

Copyright for this article is retained by the author(s), with first publication rights granted to the journal.

This is an open-access article distributed under the terms and conditions of the Creative Commons Attribution license (http://creativecommons.org/licenses/by/3.0/). 